\title{
Is Anterior-Only Fixation Adequate for Three- Column Injuries of the Cervical Spine?
}

\author{
Siddharth Sekhar Sethy, Kaustubh Ahuja, Syed Ifthekar, Bhaskar Sarkar, Pankaj Kandwal \\ Department of Orthopaedics, All India Institute of Medical Sciences, Rishikesh, India
}

\begin{abstract}
Study Design: Retrospective case series.
Purpose: To analyze the clinical and functional outcomes of patients who have undergone anterior cervical discectomy/corpectomy and fusion (ACDF/ACCF) for a three-column cervical spine injury (CSI).

Overview of Literature: The treatment of choice for a three-column CSI is an area of contention; however, combined anterior and posterior fixation is the preferred method explored in the literature. Studies have shown the superior biomechanical stability of posterior fixation over that of anterior fixation, but anterior-only approach in CSI has been proving its efficacy in recent times by providing reasonable stability with the maximum achievable decompression and fusion.

Methods: Twenty-one patients undergoing ACDF/ACCF with a bone graft/metallic cage treatment for cervical injuries involving all three columns from January 2016 to July 2018 were included in the study. All of the patients were followed up monthly for the first 3 months and then every 6 months, until their last follow-up visit.

Results: Nineteen patients had $A 0$ type $C$ injuries and were managed with $A C D F$, and two patients with $A 0$ type $B$ injuries were managed with ACCF. Fifteen had a complete spinal cord injury, while six had an incomplete spinal cord injury (American Spinal Injury Association $B, C$, and D). The mean segmental kyphosis at presentation of $12.2^{\circ} \pm 4.4^{\circ}$ improved in the postoperative period to $-7.2^{\circ} \pm 2.5^{\circ}$. At their final follow-up, all the patients showed clinical improvements when assessed by the Visual Analog Scale (6.8-1.8), Oswestry Disability Index score (59.7-34.9), and Spinal Cord Independence Measure score (24.8-36.4). One patient in the ACDF group needed a secondary posterior fixation because of instability.

Conclusions: An anterior approach to the cervical spine in cervical fracture dislocations is an effective treatment showing an optimal recovery rate in terms of patient-reported outcomes and structural stability, with the added advantages of less blood loss and the fact that the technique requires less instrumentation.
\end{abstract}

Keywords: Cervical spine dislocation; Three-column injury; Anterior-only approach; Anterior cervical discectomy/corpectomy and fusion

\section{Introduction}

Injury to the cervical spine involving all three columns is a serious problem in acute trauma scenarios that accounts for $2.4 \%$ of all blunt trauma [1] and 55\% of all spinal cord injuries [2]. Commonly presenting injury patterns involving all three columns include flexion-distraction injuries (AO type $\mathrm{B}$ ) and cervical fracture dislocations (AO type

Received Jul 9, 2019; Revised Oct 8, 2019; Accepted Oct 16, 2019

Corresponding author: Pankaj Kandwal

Department of Orthopaedics, All India Institute of Medical Sciences (AIIMS), Rishikesh-249201, Uttarakhand, India

Tel: +91-8475000282, Fax: +91-0135-2462941, E-mail: pankajkandwal27@gmail.com 
C). The most commonly reported sources of cervical spine injuries are vehicular accidents (41\%), falls (27\%), violence (15\%), sports-related injuries (8\%), and heavy objects falling onto the head [3].

Management of three-column injuries of the cervical spine (TCICS) demands achieving an adequate reduction with the reconstruction of the anterior and posterior tension bands, the decompression of neurological structures, and an emphasis on achieving early fusion for rehabilitation. Although combined anterior and posterior stabilization is considered the choice of surgery for these injuries, with improved instrumentation techniques and the right indications, anterior- or posterior-only stabilization to create instrumented fusion may prove to be an attractive alternative to combined surgery. Though biomechanical studies have shown that posterior stabilization allows for a more rigid fixation than anterior plates [4], the anterior approach allows direct decompression in cases with a protruded intervertebral disc or a fractured, retropulsed bone fragment, both of which may require the use of a large graft surface area, reducing the number of fused segments. The aim of this study is to assess the clinical and functional outcomes in patients undergoing anterior cervical discectomy/corpectomy and fusion (ACDF/ACCF) for TCICS.

\section{Materials and Methods}

\section{Patients}

This study is a retrospective case series from prospectively collected data of all the patients with a cervical flexiondistraction (AO type $\mathrm{B}$ ), or fracture dislocations (AO type $C$ ), injury surgically treated via $\mathrm{ACCF} / \mathrm{ACDF}$ in our institute from January 2016 to July 2018. The study was approved from the institutional review board of All India Institute of Medical Sciences (AIIMS/IEC/19/842). Informed consent was obtained from all individual participants included in the study. Patients with an injury at more than a single cervical level, or radiological evidence of compression over the posterior cord due to the fracture of posterior elements, were excluded from the study. Informed consent was obtained from all individual participants included in the study. The data in the registry for spinal trauma were reviewed for age, gender, mechanism of injury, level of injury, neurological status using the American Spinal Injury Association (ASIA) impairment scale, and functional status using the Visual Analog Scale (VAS), Oswestry Disability Index (ODI) score, and Spinal Cord Independence Measure (SCIM) score. Radiological data were evaluated for the type of injury, degree of subluxation/dislocation, and segmental kyphosis at the time of presentation. Facet reduction, adequate decompression, and an improvement in segmental kyphosis were evaluated in the immediate postoperative period, while bony fusion, instability, loss of correction, and implant-related complications were emphasized at the final follow-up.

All the patients who were brought in with a suspected cervical spinal cord injury were immobilized with a Philadelphia collar. Radiological data included plain radiographs in anteroposterior (AP) and lateral views, noncontrast computed tomography (CT) images, and magnetic resonance (MR) images. The criteria for a diagnosis of TCICS included radiographs and CT scans suggestive of unilateral or bilateral, jumped, or perched facets or facetal fractures (or both) and flexion-distraction type injuries. MR images provided additional information about retropulsed disc fragments, the grade of spinal cord compression, cord edema or hematoma, and the magnitude of ligamentous injury. Patients with cervical fracture dislocations underwent a closed reduction under general anesthesia (GA) in an operating theater, followed by ACDF in the same session. In patients where surgery was expected to be delayed due to poor physical condition or a lack of surgical fitness, a closed reduction was attempted with cervical traction using Crutchfield tongs, with a gradually increasing weight, followed by ACDF/ACCF once the patient was deemed fit for surgery. Two patients with AO type B2 injuries with retropulsion of a posterior fragment underwent corpectomy and anterior instrumented fusion (ACCF) (Fig. 1).

\section{Surgical technique}

All the surgeries were performed by a single, senior spine surgeon. After GA and patient positioning, a closed reduction was attempted by manual cervical traction and flexion, followed by a gradual extension of the head under intraoperative fluoroscopy. Once the reduction was achieved, the extended position of the head and neck was secured using a soft roll in the interscapular region and by strapping the forehead. In cases where a closed reduction was not achieved, we considered an open reduction after a discectomy. A standard Smith and Robinson [5] ap- 


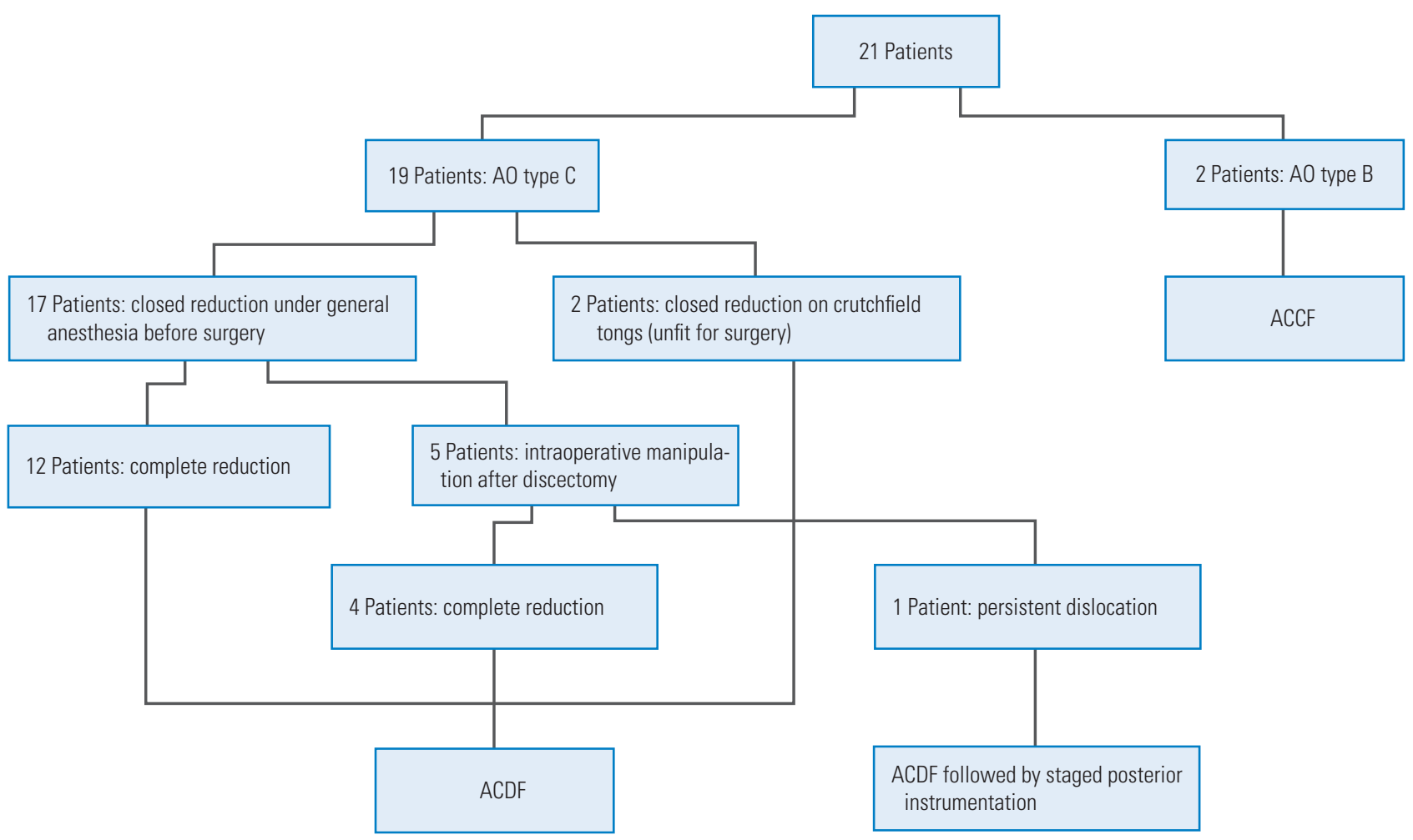

Fig. 1. Summary of management in the study group. ACCF, anterior cervical corpectomy and fusion; ACDF, anterior cervical discectomy and fusion.

proach was used for discectomies. We routinely removed posterior longitudinal ligament to examine the cord and to remove any retropulsed discs. Corpectomies were performed in cases with a vertebral body fracture and retropulsed bony fragments. Tricortical bone grafts harvested from the iliac crest, a polyether ether ketone (PEEK) interbody cage, or a mesh cage, filled with autologous bone grafts (in corpectomy cases), were used to obtain interbody fusion. A locking anterior cervical plate fixed with unicortical screws was used for stabilization. The final fixation was checked in the AP and lateral planes.

Postoperatively, X-rays and CT scans were done to confirm both reduction and fixation. Patients were kept under observation and postoperative immobilization with a sterno-occipital mandibular immobilization brace. After discharge from the hospital, they were followed up regularly at monthly intervals for 3 months and then every 6 months, until their last follow-up. Flexion and extension radiographs were done at the end of 3 months and then at 12 months, or at their last follow-up, to look for any implant-related complications, instability, or inadequate bone formation. Neurological and functional assessments were done at each follow-up, using ASIA and VAS grading, as well as ODI and SCIM scores.

\section{Results}

In retrospective data retrieved after 2 years, 28 cervical trauma patients were operated on, out of which $22 \mathrm{pa}$ tients met the inclusion criteria and were included in the study. One patient expired in the postoperative period due to pulmonary complications and was excluded from the study. The included patients had an approximate male-to-female ratio of 5:2 (15 males, six females) with a mean age of 45.3 years (range, 18 to 70 years). Out of 21 patients, 19 had AO type $\mathrm{C}$ injuries and were managed with $\mathrm{ACDF}$, and 2 patients with $\mathrm{AO}$ type $\mathrm{B}$ injuries were managed with ACCF (Table 1). The levels involved were C3-C4 (1), C4-C5 (4), C5-C6 (10), and C6-C7 (6), with C5-C6 being the most common level. The mechanism of injury was observed to be a fall from height in $14 \mathrm{pa}-$ tients $(66.6 \%)$ and road traffic accidents in seven patients (33.3\%). The average duration of the follow-up period was 16.8 months (range, 11 to 29 months). Fifteen patients had a complete cord injury (ASIA A), while six patients had an incomplete cord injury (two ASIA B, three ASIA $\mathrm{C}$, and one ASIA D). Out of 19 patients with AO type C injuries, 13 patients were found to have a bilateral facet dislocation, while six patients had a unilateral facet dislo- 

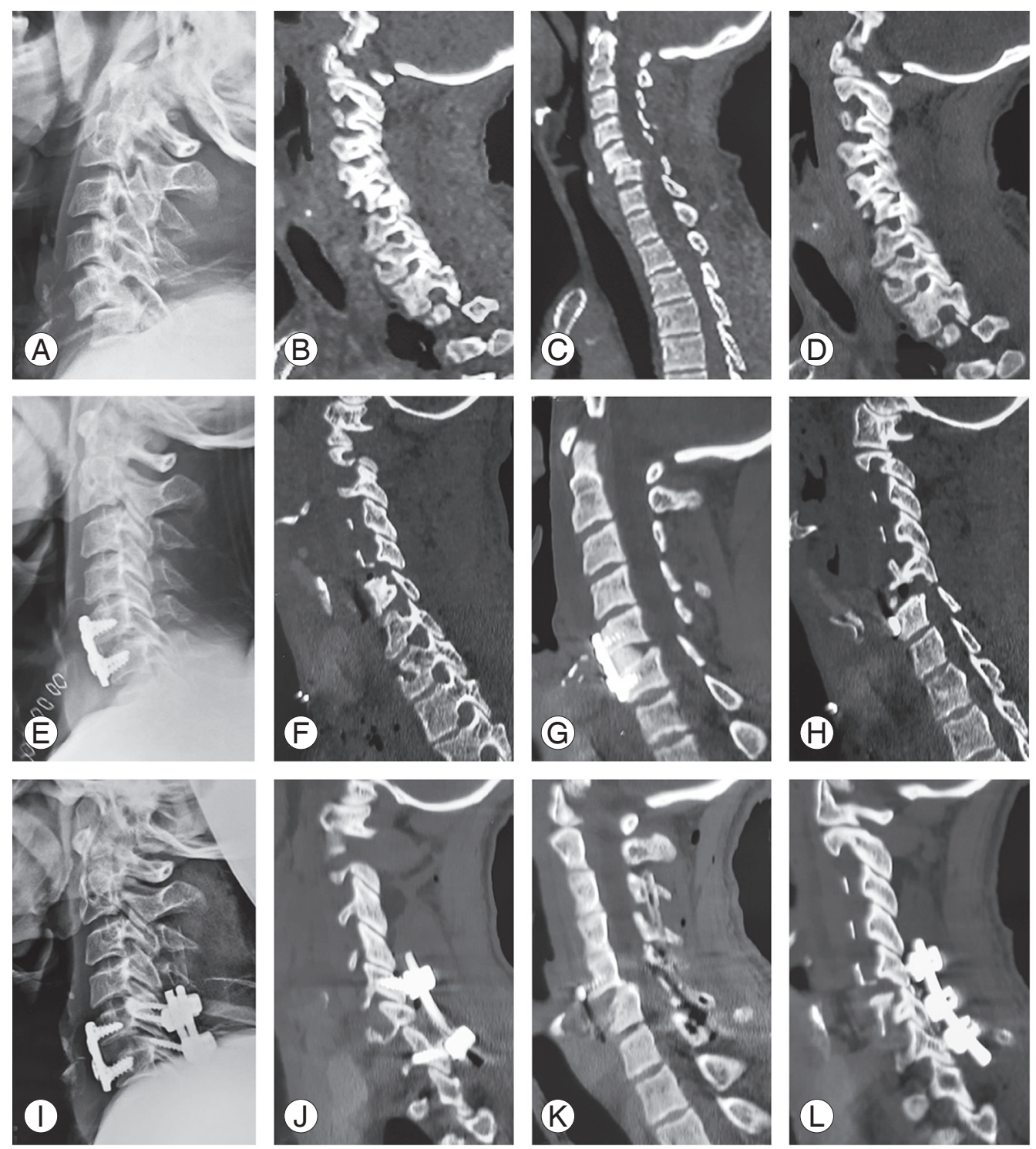

Fig. 2. Radiology of a 45-year-old male with an $A 0$ type $C$ injury. Preoperative (A) lateral $X$-ray and $C T$ images in (B) right parasagittal, (C) midsagittal, and (D) left parasagittal sections. Post anterior cervical discectomy and fusion (stage 1) (E) lateral X-ray and CT images in (F) right parasagittal, (G) midsagittal, and (H) left parasagittal sections showing persistent facet subluxation. Post posterior instrumentation (stage 2) (I) lateral X-ray and CT images in (J) right parasagittal, (K) midsagittal, and (L) left parasagittal sections with a complete reduction of facet joints. CT, computed tomography.

cation. Seventeen patients underwent a closed reduction under GA on the table before surgery, while two patients had to be put on Crutchfield tongs as their pulmonary condition needed optimization before surgery. Out of the 17 patients, a complete reduction was achieved in 12 cases, while another five needed intraoperative manipulation. In one patient, a reduction could not be achieved despite intraoperative manipulation. Staged posterior instrumentation with lateral mass screws and posterolateral fusion was done for the patient, and a complete fusion was achieved by the final follow-up (Fig. 2). Overall, 19 patients underwent ACDF with bone grafts from the iliac crest (15 patients) (Fig. 3) or a PEEK interbody cage (four patients), and two patients underwent ACCF with a mesh cage (Fig. 4).

The mean operative time was $177.3 \pm 33.8$ minutes, and the average blood loss was observed to be $253.1 \pm 109.7$ $\mathrm{mL}$. There were no intraoperative complications. Seven patients reported an improvement in neurology by 1 or 2 grades on the ASIA scale, while 14 showed no improvement (Table 2). Radiologically, the mean segmental kyphosis angle at presentation was $12.2^{\circ} \pm 4.4^{\circ}$, while, in 

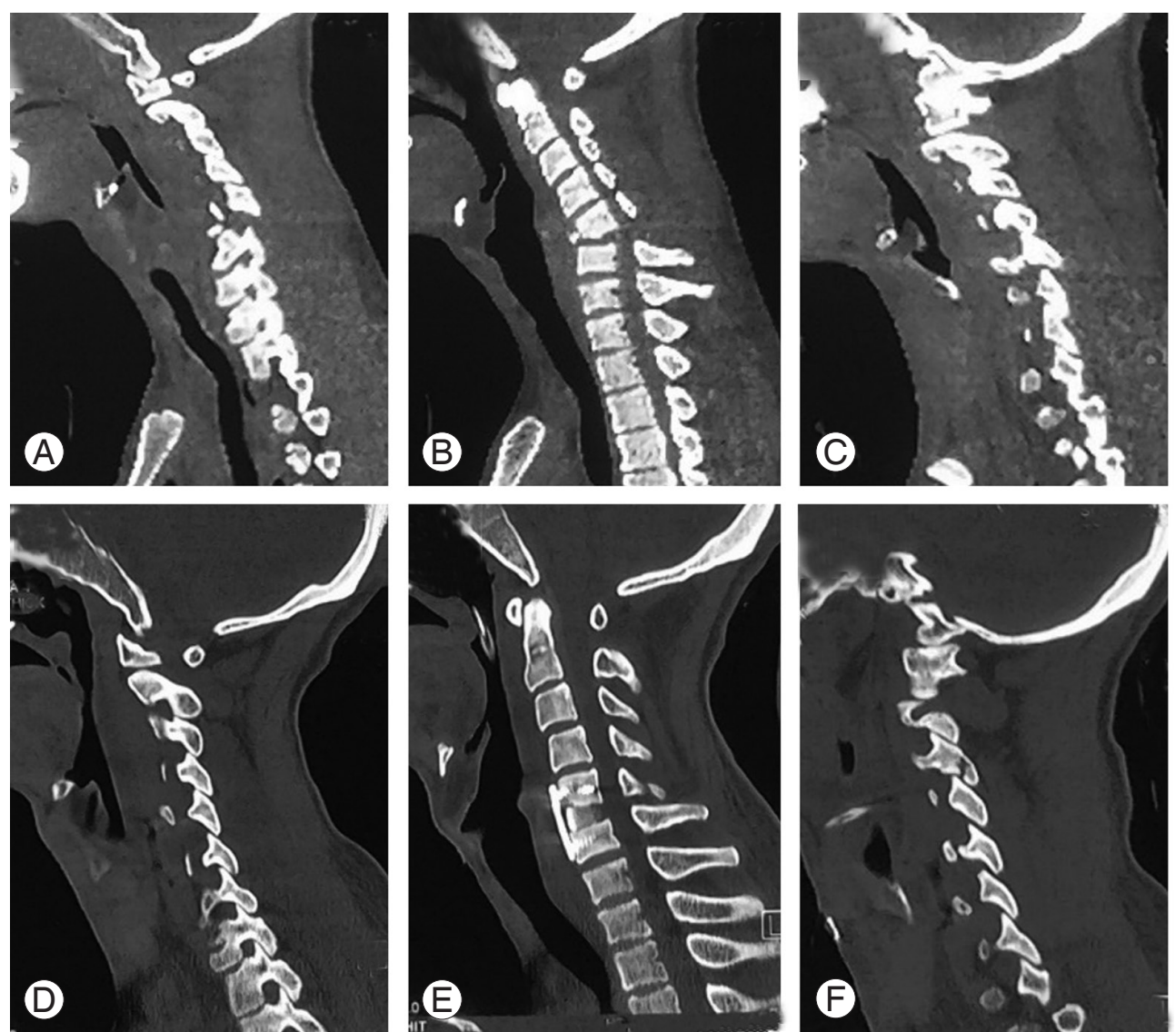

Fig. 3. Preoperative NCCT images of a 22-year-old male with an AO type $\mathrm{C}$ injury showing (A) right parasagittal, (B) midsagittal, and (C) left parasagittal sections with right facet dislocation and left facet subluxation at the C5-C6 level. Post anterior cervical discectomy and fusion NCCT images in (D) right parasagittal, (E) midsagittal, and (F) left parasagittal sections with a complete reduction of facet joints. NCCT, non-contrast computed tomography.

the immediate postoperative period, it was $-7.2^{\circ} \pm 2.5^{\circ}$ $(p<0.05)$. At the last follow-up, the mean loss of correction was $2.1^{\circ} \pm 0.9^{\circ}$. All patients showed bony fusion of the operated levels at the final follow-up. There was no evidence of instability as seen in flexion-extension radiographs at the time of last follow-up in any of the patients.

The preoperative mean VAS was $6.8 \pm 2.1$, which showed a gradual improvement that was recorded over the serial follow-ups with the final mean VAS being $1.8 \pm 1.1(p<0.05)$. The mean ODI score showed a similar trend with a mean preoperative score of $59.7 \pm 11.6$ and $34.9 \pm 13.4$ at the final follow-up $(p<0.05)$. The preoperative SCIM score was $24.8 \pm 17.2$, which improved to $36.4 \pm 23.7$ $(p<0.05)$.

\section{Discussion}

TCICS occurs as a result of hyperflexion with, or without, a rotational element to the cervical spine. The sub-axial spine is commonly involved, with nearly $70 \%$ of cases located in the C4-C7 region [6]. In our data, C5-C6 was the most common level. Falling from a height was found to be the most common mode of injury. Nineteen patients were found to have cervical fracture dislocations, while two patients had a flexion-distraction injury.

Achieving an early reduction of the dislocated facets is the first step in the management of patients with cervical fracture dislocations. Different authors have different recommendations with respect to the technique of the said reduction. A reduction of the dislocated facets can be achieved in the emergency room by the application of cervical traction [7], in the operating room under GA before surgery [8-10], or intraoperatively by distraction after a discectomy [11-13]. We achieved a reduction in $70.5 \%$ of patients (12 out of 17) by using a technique involving manipulation on the table under GA without any attempt at a closed reduction by traction; this was to avoid time loss and patient discomfort $[12,13]$. We achieved a reduction 

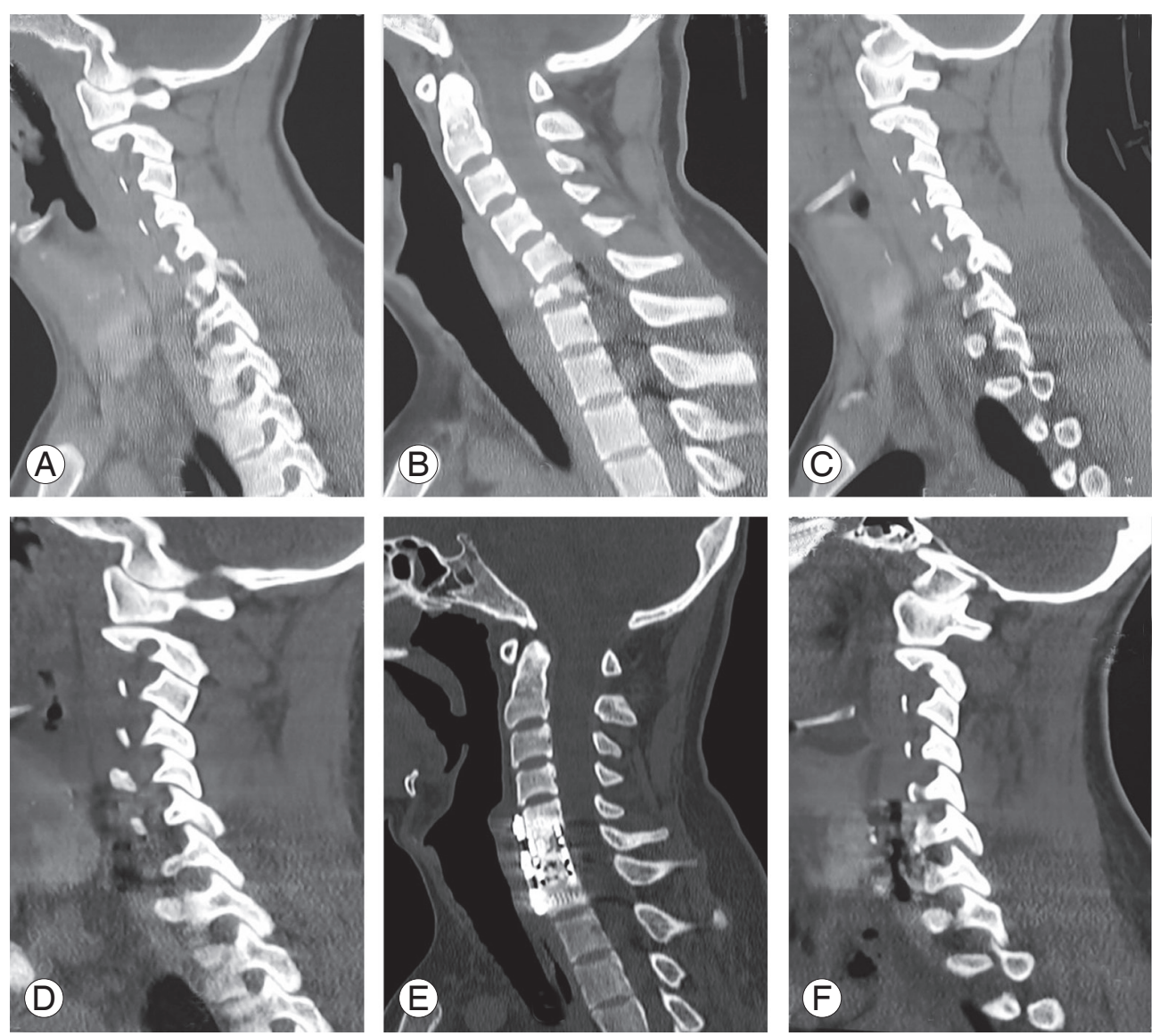

Fig. 4. Preoperative NCCT images of an 18-year-old male with an AO type B2 injury at the C6-C7 level showing (A) right parasagittal, (B) midsagittal, and (C) left parasagittal sections. Post anterior cervical corpectomy and fusion NCCT images in (D) right parasagittal, (E) midsagittal, and (F) left parasagittal sections with evident bony fusion at a 14-month follow-up. NCCT, non-contrast computed tomography.

by intraoperative distraction after a discectomy in four patients, and in one patient with persistent dislocation, a staged posterior approach was deemed necessary. All five patients with failed attempts at closed reductions had injuries more than 72 hours old, which could explain the inability to achieve a reduction by manual traction.

Being the most mobile segment in the axial spine, the need for adequate stabilization cannot be overemphasized. Optimal stabilization for TCICS remains controversial due to the lack of randomized prospective trials. From the anterior-only, posterior-only, and combined anterior and posterior stabilization techniques, the combined approach is reported to be superior in terms of biomechanical stability $[4,14,15]$. Despite the theoretical biomechanical advantage, a number of studies have reported comparable results with anterior-only fixation $[14,16]$. Anterior-only surgery has less blood loss and a shorter operative time and avoids prone turning of the patient intraoperatively, something that poses a potential risk of losing reduc- tion [7]. Facet reduction by the anterior approach can be achieved via intraoperative manipulation with a distraction technique [17] or, if required, by a facetectomy [18]. Moreover, the anterior-only approach offers direct decompression [19-22], by removing the offending agent and reducing the number of fused segments or the large surface area (i.e., the interbody area) to be fused, and has a lower infection rate [23].

The posterior approach is considered superior to the anterior approach in achieving reduction [24], but in cases with the presence of a retropulsed disc fragment, reduction by a posterior-only approach may lead to further neurological deterioration [25]. The posterior approach is reserved for cases with posterior cord compression due to fractured posterior elements or irreducible fracture dislocation with failed open, or closed, anterior reduction techniques. In our data, a staged posterior fixation was needed in one case with the dislocation persisting after an attempted closed reduction and an attempted anterior 


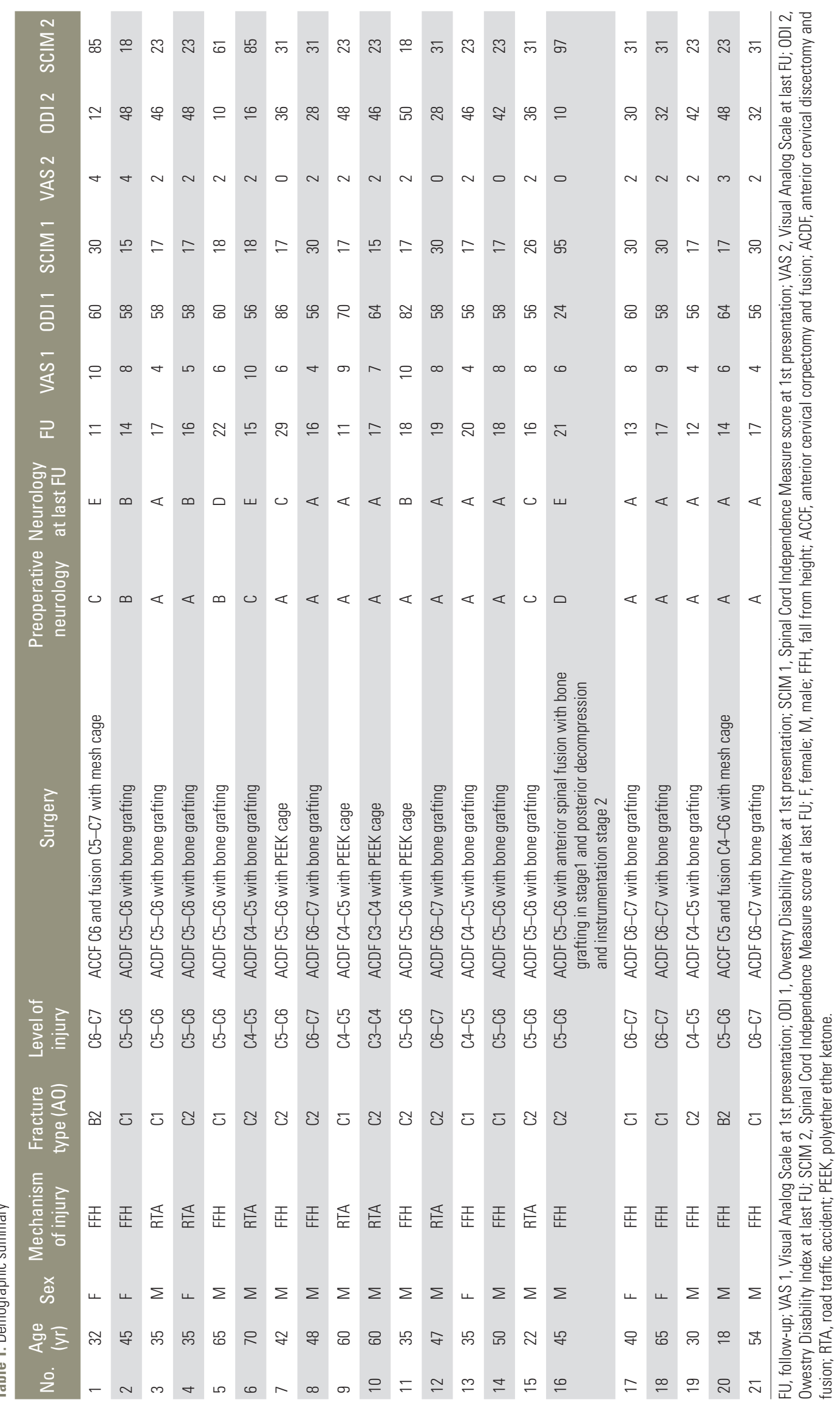


Table 2. Neurological outcome

\begin{tabular}{ll} 
Preoperative & Last follow-up \\
\hline ASIA A $(n=15)$ & ASIA A (n=12) \\
& ASIA B (n=2) \\
& ASIA C (n=1) \\
\hline ASIA B (n=2) & ASIA B (n=1) \\
\hline ASIA C $(n=3)$ & ASIA D (n=1) \\
\hline ASIA D $(n=1)$ & ASIA C (n=1) \\
\hline
\end{tabular}

reduction with stabilization.

None of the patients in our data showed a worsening of their neurology. An improvement in neurology was seen in $33.3 \%$ of patients ( 7 out of 21 ). Among the patients with an incomplete spinal cord injury, four out of six patients showed an improvement in their ASIA grade, and five out of six patients achieved an ambulatory status after surgery. A significant improvement in VAS, ODI, and SCIM scores was seen at the final follow-up in all the patients. The neurological and functional outcomes were comparable to other studies describing the management of TCICS with posterior or combined approaches [7,12-14,16]. Radiologically, the segmental kyphosis angle showed significant improvement at the final follow-up $\left(-5.1^{\circ} \pm 1.4^{\circ}\right)$ when compared to the preoperative kyphosis angle $\left(12.2^{\circ} \pm 4.4^{\circ}, p<0.05\right)$. There was a mean loss of $2.1^{\circ}$ of correction between the immediate postoperative period and the last follow-up. This can be explained due to interface bone resorption in cases that used an iliac crest bone graft or minimal cage subsidence in cases using a PEEK or mesh cage. Achieving and maintaining a reduction of the facet joints is the most important factor when determining the correction of kyphosis deformity in cases of cervical fracture dislocations. These results are comparable to other studies evaluating the radiological outcomes of surgically managed patients with cervical fracture dislocations $[14,16]$. A facet reduction was achieved in all our patients, and follow-up imaging showed bony fusion in all our cases. None of our patients had any instability or implant-related complications at the final follow-up.

This article has a few limitations: the study design being retrospective could lead to bias, the small sample size, and the short follow-up period.

\section{Conclusions}

Most patients with TCICS can be managed by anterioronly stabilization with good neurological, radiological, and functional outcomes with a minimal complication rate. Further randomized prospective studies are required for a better comparison with the combined approach.

\section{Conflict of Interest}

No potential conflict of interest relevant to this article was reported.

\section{Author Contributions}

Conception and design: SSS; data acquisition: SSS, BS, PK; analysis of data: SSS, KA, SI, BS, PK; drafting of the manuscript: SSS; critical revision, KA, SI; supervision: KA, SI, BS, PK; and administrative support: BS, PK

\section{References}

1. Goldberg W, Mueller C, Panacek E, et al. Distribution and patterns of blunt traumatic cervical spine injury. Ann Emerg Med 2001;38:17-21.

2. Sekhon LH, Fehlings MG. Epidemiology, demographics, and pathophysiology of acute spinal cord injury. Spine (Phila Pa 1976) 2001;26(24 Suppl):S2-12.

3. Burke DA, Linden RD, Zhang YP, Maiste AC, Shields CB. Incidence rates and populations at risk for spinal cord injury: a regional study. Spinal Cord 2001;39:274-8.

4. Do Koh Y, Lim TH, Won You J, Eck J, An HS. A biomechanical comparison of modern anterior and posterior plate fixation of the cervical spine. Spine (Phila Pa 1976) 2001;26:15-21.

5. Smith GW, Robinson RA. The treatment of certain cervical-spine disorders by anterior removal of the intervertebral disc and interbody fusion. J Bone Joint Surg Am 1958;40-A:607-24.

6. Aebi M. Surgical treatment of upper, middle and lower cervical injuries and non-unions by anterior procedures. Eur Spine J 2010;19 Suppl 1:S33-9.

7. Razack N, Green BA, Levi AD. The management of traumatic cervical bilateral facet fracture-dislocations with unicortical anterior plates. J Spinal Disord 
2000;13:374-81.

8. Lu K, Lee TC, Chen HJ. Closed reduction of bilateral locked facets of the cervical spine under general anaesthesia. Acta Neurochir (Wien) 1998;140:1055-61.

9. Doran SE, Papadopoulos SM, Ducker TB, Lillehei KO. Magnetic resonance imaging documentation of coexistent traumatic locked facets of the cervical spine and disc herniation. J Neurosurg 1993;79:3415.

10. Mahale YJ, Silver JR, Henderson NJ. Neurological complications of the reduction of cervical spine dislocations. J Bone Joint Surg Br 1993;75:403-9.

11. Anissipour AK, Agel J, Baron M, Magnusson E, Bellabarba C, Bransford RJ. Traumatic cervical unilateral and bilateral facet dislocations treated with anterior cervical discectomy and fusion has a low failure rate. Global Spine J 2017;7:110-5.

12. Payer M. Immediate open anterior reduction and antero-posterior fixation/fusion for bilateral cervical locked facets. Acta Neurochir (Wien) 2005;147:50913.

13. Ordonez BJ, Benzel EC, Naderi S, Weller SJ. Cervical facet dislocation: techniques for ventral reduction and stabilization. J Neurosurg 2000;92(1 Sup$\mathrm{pl)}: 18-23$.

14. Kim SM, Lim TJ, Paterno J, Park J, Kim DH. A biomechanical comparison of three surgical approaches in bilateral subaxial cervical facet dislocation. J Neurosurg Spine 2004;1:108-15.

15. Coe JD, Warden KE, Sutterlin CE 3rd, McAfee PC. Biomechanical evaluation of cervical spinal stabilization methods in a human cadaveric model. Spine (Phila Pa 1976) 1989;14:1122-31.

16. Kim KH, Cho DC, Sung JK. The management of bilateral interfacetal dislocation with anterior fixation in cervical spine: comparison with combined antero-posterior fixation. J Korean Neurosurg Soc 2007;42:305-10.

17. Kanna RM, Shetty AP, Rajasekaran S. Modified anterior-only reduction and fixation for traumatic cervical facet dislocation (AO type C injuries). Eur Spine J 2018;27:1447-53.

18. Liu K, Zhang Z. Comparison of a novel anterior-only approach and the conventional posterior-anterior approach for cervical facet dislocation: a retrospective study. Eur Spine J 2019;28:2380-9.

19. Laus M, Pignatti G, Tigani D, Alfonso C, Giunti A. Anterior decompression and plate fixation in fracture dislocations of the lower cervical spine. Eur Spine J 1993;2:82-8.

20. Cloward RB. Treatment of acute fractures and fracture-dislocations of the cervical spine by vertebralbody fusion: a report of eleven cases. J Neurosurg 1961;18:201-9.

21. Reindl R, Ouellet J, Harvey EJ, Berry G, Arlet V. Anterior reduction for cervical spine dislocation. Spine (Phila Pa 1976) 2006;31:648-52.

22. De Oliveira JC. Anterior reduction of interlocking facets in the lower cervical spine. Spine (Phila Pa 1976) 1979;4:195-202.

23. Levi AD, Dickman CA, Sonntag VK. Management of postoperative infections after spinal instrumentation. J Neurosurg 1997;86:975-80.

24. Park JH, Roh SW, Rhim SC. A single-stage posterior approach with open reduction and pedicle screw fixation in subaxial cervical facet dislocations. J Neurosurg Spine 2015;23:35-41.

25. Nakashima H, Yukawa Y, Ito K, Machino M, El Zahlawy H, Kato F. Posterior approach for cervical fracture-dislocations with traumatic disc herniation. Eur Spine J 2011;20:387-94. 